Mots. Les langages du politique

$70 \mid 2002$

La politique en chansons

\title{
Rap et politique
}

Rap and Politics

Rap y política

Vincent Fayolle et Adeline Masson-Floch

\section{(2) OpenEdition}

Journals

Édition électronique

URL : https://journals.openedition.org/mots/9533

DOI : $10.4000 /$ mots.9533

ISSN : 1960-6001

Éditeur

ENS Éditions

Édition imprimée

Date de publication : 1 novembre 2002

Pagination : 79-99

ISBN : 2-84788-016-X

ISSN : 0243-6450

\section{Référence électronique}

Vincent Fayolle et Adeline Masson-Floch, « Rap et politique », Mots. Les langages du politique [En ligne], 70 | 2002, mis en ligne le 07 mai 2008, consulté le 22 avril 2022. URL : http://journals.openedition.org/ mots/9533 ; DOl : https://doi.org/10.4000/mots.9533 


\section{Vincent FAYOLLE ${ }^{\circ}$}

Adeline MASSON-FLOCH ${ }^{\circ}$

\section{Rap et politique}

Nous aborderons la question de la portée politique du rap à travers l'analyse de la complexité des foyers énonciatifs ${ }^{1}$ qui peuvent être mobilisés, modifiés ou initiés dans le cours des textes. La cohérence de ces foyers conduit à poser la question des identités construites et véhiculées au sein de ce discours, selon un mode de diffraction qui met en cause le concept de communauté, tant du point de vue énonciatif que du point de vue de l'espace de destination qui lui est corrélatif.

Une fois défini le cadre général dans lequel nous envisageons l'inscription critique du mouvement et du discours rap au sein du champ politique, nous dégagerons les caractéristiques de l'engagement complexe que le rap fait percevoir, à partir du corpus que nous avons recueilli en 2002 à Draveil dans l'Essonne ${ }^{2}$, et nous mettrons l'accent sur la portée politique propre aux diverses mises en mots.

- GRAFEC, Université Paris 5 - René Descartes.

1. On parlera ici de l'énonciation représentée, c'est-à-dire telle qu'on peut l'analyser à partir de textes.

2. Le GRAFEC (Groupe de Recherche Appliquée aux Formes d'Expression Contemporaines), créé en janvier 2000, est co-dirigé par Michelle Auzanneau et Margaret Bento. Il participe à l'analyse pluridisciplinaire de données récoltées par M. Auzanneau au Sénégal et au Gabon dans le cadre d'une recherche sur le rap africain entamée en 1999-2000. Dans le prolongement de cette étude, nous réalisons actuellement une enquête auprès de jeunes rapeurs vivant en banlieue parisienne (Essonne). Recherche de terrain qui s'intéresse à certains aspects et manifestations du rap français, et dont sont issues les données qui constituent la base de référence et d'illustration de la réflexion menée au cours de cet article. 


\section{Le rap : prise de parole d'une jeunesse citadine}

Le rap constitue une prise de parole publique, fortement contextualisée sur le plan social et qui a pour vocation d'être diffusée. Il nous apparait comme un évènement artistique et identitaire ${ }^{3}$ fondamentalement politique. Le discours rap nous semble constituer en lui-même un mode et un champ de positionnements vis-à-vis de l'ensemble des domaines du politique 4 apparaissant dans sa définition traditionnelle (« cité », "État», « société », «pouvoir»). Mais il use le plus souvent de stratégies de contournement ou de détournement (plus ou moins conscientes et implicites) de ces instances et institutions légitimes et légitimantes, à commencer par la langue.

\section{La posture du rappeur : le refus des modèles dominants}

L'ensemble des études concernant le rap ${ }^{5} \mathrm{~s}$ 'accordent à le considérer comme une forme d'expression ou de « parole $\rangle^{6}$ spécifiquement urbaine, comme « un mouvement qui doit pouvoir nous renseigner sur les valeurs véhiculées en milieu urbain et, plus particulièrement au sein de la jeunesse $\gg{ }^{7}$. Le rap possède donc une double caractéristique. L'espace citadin se présente comme le tissu social complexe dont émerge la parole rap - ou, sans doute, plutôt le concert de paroles rap. Aujourd'hui, à l'échelle internationale, plusieurs générations d'une partie de la jeunesse urbaine s'approprient cette parole et participent à son élaboration continue, selon des modèles et des codes partagés, ainsi que selon des modalités contextuellement définies par chacun de ses représentants.

Ainsi, l'émergence du rap s'est partout produite selon un rapport étroit

3. Au sens où le discours rap est le lieu de construction et d'expression d'identités complexes, face à un discours dominant, considéré comme assimilateur et réducteur.

4. L'adjectif politique désigne ce qui est « relatif à la cité, au gouvernement de l'État (...) à la société organisée (...) à l'organisation et à l'exercice du pouvoir (...) et à la lutte autour du pouvoir » (Petit Robert, édition de 1981). Il ne s'agit pas ici de nous étendre sur la valeur sémantique de ce terme, en vue de créer des rapports simplificateurs (d'opposition ou d'assimilation) entre des espaces ou formes d'engagement politiques et les contextes socio-culturels, les activités ou expressions qui caractérisent le mouvement hip-hop. Il s'agit encore moins de considérer le rap comme une démarche politique instituée, ce qui relèverait d'un contresens.

5. Études sociologiques, anthropologiques, sociolinguistiques, musicologiques... voir la bibliographie.

6. M. Auzanneau (2001a) parle de « parole urbaine », p. 711.

7. M. Boucher, 1998, p. 23. 
et affirmé au phénomène urbain, à son essor, à sa gestion, et aux configurations sociales auxquelles il a donné lieu. «La ville qui tend à une certaine uniformisation des comportements s'offre aussi comme un lieu de distinctions sociales $»^{8}$. Elle est, par excellence, le point de convergence de flux et de réseaux migratoires divers, et diversement motivés. Elle est l'espace de vie, d'expression et de contact de cultures, de langues, et de communautés variées, qui vont dès lors se co-définir les unes par rapport aux autres, mais aussi se re-définir de l'intérieur du fait d'une appartenance recomposée, plurielle. Dans ce cadre, les positionnements sociaux variables des individus, négociés en contexte, expriment des identités citadines multiples ${ }^{9}$. Ces négociations s'effectuent selon les paramètres des situations et interactions où les individus sont engagés (interlocuteur(s), objet(s) et objectif(s) des échanges de diverses natures, etc.), ainsi qu'en référence à un ou plusieurs réseaux sociaux ${ }^{10}$ dans lesquels ils s'insèrent, à l'hétérogénéité de ceux-ci, ou enfin aux spécificités de leurs parcours. Nous considérons que «la variation des pratiques linguistiques reflète cette mouvance et cette pluralité culturelle et sociale ${ }^{11}$. Nous nous appuyons sur un ensemble d'hypothèses selon lesquelles la chanson révèle certains aspects des dynamiques sociolinguistiques en cours dans ces contextes, en même temps qu'elle participe à l'élaboration et à la mise en circulation d'usages, de représentations et de marques linguistiques ${ }^{12}$.

Les choix langagiers opérés dans l'ensemble des textes de rap sénégalais, gabonais et français sur lesquels nous travaillons, attestent de la prise en charge par leurs auteurs des différents aspects de la problématique urbaine; tant du point de vue de leurs manifestations quotidiennes, que de leur dimension socio-historique et de leur résonance politique. Leur cadre de vie citadin, et plus largement les rapports de force sociaux qui s'y jouent de façon accrue ${ }^{13}$, constituent la source d'inspiration majeure des rappeurs ainsi que le moteur de leur (ré)activité énonciative et réflexive investie dans le rap. La majorité des chansons fait explicitement référence à ce contexte à travers la mise en scène ou l'évocation des réalités et pra-

8. M. Auzanneau, 2001a, p. 711.

9. Et de façon plus nuancée, des quêtes ou gestes identitaires divers.

10. Au sens de « social networks » définis par L. \& J. Milroy, 1990.

11. M. Auzanneau, M. Bento, V. Fayolle, 2002, p. 70.

12. Nos analyses envisagent aussi de prendre en compte les contraintes formelles et esthétiques du genre, qui impliquent une rencontre entre rythme linguistique et discursif, rythme musical et métrique. Ces aspects ne seront pas développés ici.

13. Rapports (au sens de liens et non-liens sociaux) de classe, de sexe, de culture, de génération, de fonction et de rôle, etc. 
tiques quotidiennes liées à la vie de la rue, du quartier, du ghetto, de la cité, dont les rappeurs (individuellement et collectivement) témoignent, auxquelles ils prennent part et/ou sur lesquelles ils s'interrogent.

1- Je tchatche des réalités $100 \%$ réalités / si tu ne veux pas me croire regarde dehors la réalité / si j'esquive la réalité bien je suis un vendu / je suis un de ces gars qui baissent les yeux devant les problèmes de la rue ${ }^{14}$. (Beozedzed, Je suis dans mon block, je rap)

2- Toujours la même tess ${ }^{15}$ / toujours les mêmes bizness / toujours le même stress / [...] Trop de rage! Comment survivre en ville sans vivre dans le vice? (JK, Quotidien)

Les rappeurs peuvent alors s'ériger en « représentants » d'espaces clairement localisés et d'un des groupes sociaux au moins qui y évoluent, espaces et groupes auxquels ils revendiquent ainsi leur appartenance.

3- Contre les fachos je préfère prendre le micro / représenter tous mes tepos / Athis Mons 9.1.2.0.0 / c'est comme ça que je fais sur le tempo / [...] Allez allons-y représentons nous aussi pour nos possee ${ }^{16}$. (Beozedzed, Je suis dans mon block, je rap)

4- JK d'la RAD investit dans l'son jusqu'à la nuque / Kainfindies ${ }^{17}$ dans l'sang envoie ta voix avant qu'on t'nique / 91100 représentant de Corbeil à Saint Michel / l'union fait la force pour s'imiscer en haut de l'échelle / KF dans l'son, KF dans l'sang / KF du 91100 à St Michel en Essone. (JK, Made in A ...)

Selon M. Boucher, « le mouvement rassemble toute une jeunesse des banlieues et des quartiers défavorisés ainsi que la jeunesse des classes moyennes en quête d'une identité nouvelle et plurielle $\gg{ }^{18}$. Cette appartenance à des milieux ou communautés décrits comme négligés, minorés, voire marginalisés ou stigmatisés, ou qui plus généralement ne sont pas reconnus dans leur diversité, leur richesse et leurs difficultés propres,

14. N. B. : nous avons préservé l'orthographe originale des textes.

15. Tess : troncation de téci, verlan de cité.

16. Possee : terme issu du lexique rap américain, qui signifie « groupe, clan ».

17. Kainfindies $(K F)$ : mot-valise, composé de kainf: troncation par apocope du verlan de africain (cainfri), et de indies : aphérèse de West Indies (désignation anglophone des Antilles). Il s'agit d'un collectif de jeunes de l'Essonne, originaires d'Afrique ou des Antilles, qui souhaitent mener une action commune pour la reconnaissance de leurs origines.

18. M. Boucher, 1998, p. 196. 
semble fonder la nécessité d'une prise de parole. Celle-ci détermine la posture du rappeur comme porteur d'un message ou d'une mission, et l'autorise ainsi à fortement valoriser son acte de parole, son agilité verbale ou encore son identité de rappeur (son nom ou blaze).

5- Appelle le tiequar dis leur qu'en 2001 on fait le constat / Béozedzed et JK on tchatche sur l'instrumental / des lyrics ieuser ${ }^{19}$ comme ce que nous apprennent nos pères et mères. (Beozedzed et $\mathrm{JK}$, Descendant d'esclave)

6- Si je prends le cromi t'as vu c'est pas pour dire des conneries / moi je viens avec ma vision des choses comme beaucoup d'autres $\mathrm{MC}^{20}$ / approche, écoute, hoche la tête, j'apporte que des choses proches / branche tes potes ils verront qu'au microphone rien je cache / un mioche ou pas je balance comme ça se passe dans la place / du réel qui sort des basses / l'ami c'est comme ça que je froisse / pas de thème, moi je sais juste qu'il y a des nouvelles / et rien de nouveau au dientiquo / compte-rendu de la part de zedzedbéo. (Beozedzed, Quotidien)

7- J'ai quasiment tout dit!? / sauf ce qui se passe derrière mon dos! / Ici c'est Bastac, à mes côtés JK, FlyDchir, Beozedzed / $4 \mathrm{MC}$ qui déchirent dans leur ligne de mire!! (Bastac, Quotidien)

Dès lors son rôle revient à combler et compenser d'une part, le défaut de reconnaissance (sociale, économique, culturelle, historique...) et l'ensemble des manquements, inégalités et injustices dont ces milieux sont victimes plus que d'autres et, d'autre part, le déficit d'information ou d'éducation relatif à ces réalités sociales, à certaines de leurs causes et implications, qui concerne l'ensemble des acteurs sociaux.

8- Attiser les haines c'est pas ce qu'on cherche dans ce thème / on se doit de raconter quand même / puisque le système scolaire nous enseigne que le tiers / en France cette story on la calcule guère / donc passe le message pour qu'il se prolifère jusqu'aux futurs gosses / on vit dans un pays qui jadis jugeait nos arrière-reups / se prenait pour le seigneur mais n'était que le diable en quelque sorte. (Beozedzed, Descendant d'esclave)

9- Dans l'quotidien en France / faut voir la vie en face / il faut que ça passe ou ça casse / dans le Parisien on passe / jamais la même chance / toujours la même

19. Ieuser : verlan de sérieux.

20. $M C$ : terme issu du lexique rap américain, Master of Ceremony, qui désigne le rappeur. 
chiasse / [...] Trop de rage! Quand je vois mon pote tabassé par les shtars ${ }^{21} /$ [...] Trop de rage! Ils médiatisent que les aspects négatifs. (JK, Quotidien)

10- Et ça me fout un goût amer / de savoir qu'on est pas sur notre terre / ce qui veut dire qu'on doit se taire / parce que nos paroles sont prises à la légère / j'parle car on est tous dans la même galère / demande à ton père / les mêmes 6 -8 heures, de quoi devenir fou / fouttre le camps d'ici c'est ce qu'il me dit / Paris il m'a dit que pour lui c'était un bon bizness / laisse tomber maintenant il fait que ramer / en tant qu'immigré en France j'ai constaté que c'était pas gagné / fier de ce qu'on est même si on l'a pas demandé / combattre et pas se laisser faire / [...] ouais ouais en $2002 \mathrm{j}$ 'peux pas me taire. (Beozedzed, $X X X$ )

Plus profondément, l'ancrage du rap dans les contradictions et les violences réelles ou symboliques des sociétés urbaines modernes semble légitimer les prises de position radicales et hostiles des rappeurs à l'égard de tous les organes de domination et de pouvoir (individus, institutions) considérés comme « responsables » de cette situation, à l'égard aussi de tous les déviants internes à leurs propres groupes de référence.

11- Rebellion le blanc au sommet n'a jamais voulu se faire égaler / [...] Rebellion parce que le système nous a trop saoulés / Rebellion maintenant il faut se rassembler et l'État doit trembler / Rebellion le FN est ciblé et Le Pen va tomber / [...] libres mais pas égaux problème qu'il faut régler / [...] Rebellion car on a pas encore de chaine de noir à la télé. (JK, Rebellion )

12- La même devise pour tous et on le sait tous / sauf les lèches qui percent et qui se disent vrais / fiers de leurs actions, toujours des exceptions et partout des corruptions / donc je me dois de faire des revendications pour q'ils sachent que je ne suis pas con. (Beozedzed, Je suis dans mon block, je rap)

Dans le même temps, cet ancrage légitime la mobilisation, voire la glorification, de toutes formes d'argumentation, de réaction et de provocation possibles, révélatrices de l'impossibilité et du refus d'intégrer le modèle dominant.

13- Rebellion parce que pour se faire entendre il faut gueuler / [...] Rebellion parce qu'il y a plein d'hommes d'État qui sont cinglés / je lève mon doigt devant l'immunité soit disant immaculée / tous les mecs au-dessus des lois qu'ils aillent se faire enculer / désolé mais je te rappelle que je suis pas venu pour rigoler / et j'en place une pour mon peuple qu'on a volé / il est temps de

21. Shtars : terme argotique désignant les policiers. 
passer à l'assaut remonte tes ourlets / la seule hymne nationale sera de t'entendre hurler / Rebellion celui qui n'est pas des notres il a qu'à brûler / parce que si on lève tout le peuple il faut pas simuler. (JK, Rebellion)

14- Trop de rage! C'est l'instinct que j'ai dégagé! / Trop de rage! Quand leur politique nous menace de dégager! / Trop de rage! Dans ma rangée on s'est engagé à tous les déranger! / Trop de rage! Quand Mégret met de son plein gré pour qu'on ait des regrès sur notre couleur! / De ma bouche au micro, du micro aux enceintes / j'veux qu'il ressente ce que je dis! / reste mal à l'aise comme une femme enceinte! / Soyez maudits! / Quand il balance des mouches aux aveugles / et louches sont les conneries qu'il dit aux sourds / reste pas abasourdi par c'que $\mathrm{j}$ ' dis frère! / on dirait que tu gobes les mouches / bouge-toi le cul, souviens-toi qu'ils abattent les gens dans le dos! (Bastac, Quotidien)

15- Jour pour jour, cas par cas / à $100 \%$ je suis condamné / d'rimes sur papier / écrit en gros « enragé 》/ voilà ma peine, voilà mon texte / même contexte devant mes Corn Flakes / j'constate les mêmes bavards, les mêmes bavures / les mêmes qui bavent / les mêmes baba flex baissent leur froc / FUCK THE MYTHO MOVEMENT / si tu veux test. (FlyDchir, Quotidien)

\section{La langue du rap, ou la voix d'une revendication}

Discours insultants, crus, métaphoriques, teintés d'humour, de fatalité ou appels à l'action, ces prises de position sont des témoignages à plusieurs faces qui oscillent entre dénonciation, révolte (discours antiflics, anti-racistes, anti-politique, anti-conformistes, etc.), revendication, conscientisation ou moralisation (discours ethnique, pro-black par exemple, informatif, pédagogique, parfois politique (groupes comme Assassin) ou religieux, etc.); qui aspirent à l'élaboration et la reconnaissance d'une identité à la fois particulière et plurielle. Ces stratégies et mouvements discursifs sont interdépendants des points de vue illustrés et des effets recherchés. De même, les usages et la créativité linguistiques participent de la richesse des positionnements et de la pertinence des actes identitaires manifestés au sein de ces discours. Dans et par la langue s'exprime une double revendication (au moins).

En premier lieu, celle d'habiles manipulateurs du langage, de ses fonctionnalités et de ses ressources expressives, esthétiques, ludiques... Les rappeurs démontrent ainsi leur compétence et leur efficacité langagières en contexte, ainsi que la coloration multilingue de leurs répertoires. En outre, la forme hétéroclite et le contenu percutant de ce discours forcent l'attention des auditeurs, tant en situation d'improvisation que de composition plus classique. 
La seconde revendication est emblématique de l'œuvre de crypteurslocuteurs qui déconstruisent la dimension sociale de la langue en tant que vecteur de communication partagé par tous les membres de la communauté attachée à la pratique courante de cette langue. En l'imprégnant de leur diversité et de leur différence, les rappeurs (re)-construisent et renvoient au public un discours marqué par les divisions sociales (la « fracture »!) qui en constituent le fondement.

En accord avec R. Nicolaï ${ }^{22}$, nous considérons que « la nature feuilletée du répertoire » permet aux rappeurs « la refonctionnalisation [dans leur discours] de traits, de formes linguistiques et de fragments discursifs et attitudinaux matériellement disponibles » dans leurs répertoires. Ces unités « refonctionnalisées », peuvent être lexicales, morpho-syntaxiques, phonologiques, prosodiques, gestuelles, etc. Leur mobilisation est corrélative de la dynamique ainsi que de la négociation de « droits et obligations réciproques $»{ }^{23}$ caractéristiques « des constructions communautaires et/ou identitaires » élaborées par les rappeurs. Leur agencement est lié, en outre, aux contraintes rythmiques et stylistiques propres au genre musical. Ces opérations contribuent à construire le sens et la portée des codes et usages langagiers "ré-élaborés » au sein du discours rap, et plus largement au sein de la jeunesse qui, à divers degrés, se reconnait dans le mouvement hip-hop. Ces codes et usages constituent ainsi un ensemble de traits et de référents communs, qui comme le suggère J. Billiez peuvent prendre la dimension d' " indicateurs identitaires ${ }^{24}$, révélateurs d'attitudes et de valeurs partagées. Dimension d'autant plus manifeste à mesure qu'ils deviennent difficilement accessibles aux non-initiés. Ainsi la langue prend toute sa valeur d'objet symbolique et social, reflet de la complexité, de la variabilité et des déséquilibres des rapports sociaux.

22. Références à R. Nicolaï, 2000. Il définit ainsi la notion de feuilletage : « Le répertoire des codes linguistiques, par exemple, possède ce caractère particulier de pouvoir fonctionner, en contexte, comme ressource dans la ré-élaboration continue de variétés linguistiques et d'usages langagiers constitués à travers la refonctionnalisation de traits, de formes linguistiques et de fragments discursifs et attitudinaux matériellement disponibles. Je parlerai de " feuilletage » à propos de cette dynamique qui permet de reconnaitre/nommer la multiplicité des usages et des variétés disponibles dans le répertoire et d'appréhender leur superposition sans pour autant leur attribuer d'homogénéité structurelle a priori ...» (p. 413).

23. Au sens où C. Myers-Scotton (1983) parle de «rights and obligations between speakers ».

24. J. Billiez, 1998, p. 138. 
Solidarités, contextes : le rap et ses espaces de légitimation

Bien que d'ordre assez général, les remarques qui précèdent, illustrées par les extraits de chansons, manifestent ce que L.J. Calvet considère comme « l'affirmation de deux types de solidarité ${ }^{25}$ dans le rap. En premier lieu, les chansons citées, par le simple fait de se donner à entendre ${ }^{26}$ comme " parole rap », se positionnent dans le vaste champ de la culture hip-hop. Elles le font à travers l'usage systématisé et combiné d'un certain nombre de composantes caractéristiques voire stéréotypiques du genre, largement empruntées au modèle américain ( «speech acts patterns » ${ }^{27}$, lexique, rythme, prosodie ou «flow», etc.). Elles s'inscrivent ainsi dans un mouvement de contestation global à l'égard de l'ensemble des instances de domination légitimes. Partout en effet le rap semble s'ancrer dans des formes de $"$ culture interstitielle ${ }^{28}$, qui s'élaborent au creux de dialectiques socio-économiques et historiques perçues comme contradictoires, inéquitables ou conflictuelles (dominants / dominés; espaces, cultures et langues d'origine / d'accueil; tradition / modernité...). L'expression de cette culture et de cette conscience commune dépasse largement le cadre de la chanson et se manifeste à travers diverses formes artistiques (DJing, danse, graff), ou encore à travers le style vestimentaire.

Toutefois, si à l'échelle internationale le rap reconnait et porte la marque d'une filiation commune dans le mouvement né aux USA à la fin des années 1970, ce sont les réalités vécues localement par les rappeurs qui en fondent la pertinence et la véracité. Ainsi s'opère au sein de chacune de ces réalités « un processus de recontextualisation $"{ }^{29} \mathrm{du}$ discours rap, tant sur le plan des thématiques développées, que sur le plan des formes linguistiques mobilisées ou créées. Cette profonde imprégnation du rap par les environnements sociaux de ses acteurs génère l'expression d'une seconde solidarité dont l'extension est extrêmement variable. Solidarité envers le groupe de pairs, le quartier, la famille, la communauté, etc., et, au sein du rap français dont de nombreux représentants sont issus des migrations, envers les pays et langues d'origine.

Du point de vue de l'expression de ces solidarités, notre terrain d'enquête en région parisienne et les textes qui y ont été recueillis, constituent

25. L.J. Calvet, 1996, p. 277.

26. Ici simplement à lire partiellement!

27. Au sens de J. Androutsopoulos et A. Scholz, 1999.

28. L.J. Calvet, 1996, p. 269, qui emprunte la notion d'« interstice » à l'École de Chicago.

29. «A recontextualisation process », J. Androutsopoulos et A. Scholz, 1999, p. 1. 
un cadre d'analyse particulièrement intéressant. Depuis plus d'un an, nous rencontrons régulièrement de jeunes rappeurs de l'Essonne (91) pris en charge dans leurs activités de composition (textes et musiques), d'enregistrement et de préparation scénique, dans le cadre d'un atelier ouvert proposé au sein d'un foyer d'accueil de la Protection Judiciaire de la Jeunesse, à Draveil. En juillet 2001, ces activités ont donné lieu à une rencontre entre sept jeunes rappeurs français (dont une chanteuse) et douze de leurs homologues sénégalais, à Dakar. L'objectif était double. Éducatif, culturel et artistique d'une part, puisqu'il s'agissait d'apprendre à vivre et à travailler ensemble afin de participer à la réalisation d'une mix-tape (ou compilation), qui serait le fruit de leur diversité et de leurs échanges ${ }^{30}$. Objectif plus largement «politique » d'autre part, dans la perspective de favoriser des échanges Nord-Sud équitables et réciproques.

Ainsi, les thèmes des chansons sur lesquelles repose le présent travail (l'esclavage, le quotidien, le racisme) ont été déterminés de part et d'autre par les jeunes Français et Sénégalais dans le but de confronter leurs témoignages, leurs expériences et leurs points de vue au sein de compositions communes. Ici, nous ne nous attachons pas spécifiquement à l'étude des chansons composées dans le seul cadre de cette rencontre ${ }^{31}$. Toutefois, notre corpus est constitué de textes s'inscrivant dans les mêmes thématiques. La dimension transversale de ces dernières est d'ailleurs confirmée par l'importance de leur traitement dans l'ensemble de nos corpus sénégalais et gabonais. Suite à nos échanges avec les jeunes Français à leur retour du Sénégal, et à la lecture des textes composés ou révisés à Dakar, nous avons perçu une évolution sensible de leur attitude vis-à-vis des « réalités africaines » (passage d'un discours interrogateur et mystificateur, à des récits ancrés dans les réalités vécues et observées). Cette évolution s'est traduite de diverses manières dans les chansons :

- par une référence explicite à l'expérience du voyage, pour exemple ces extraits de Peuple noir, texte de la chanteuse Dyara (ex-Asya), d'origine antillaise, qui a depuis adopté comme pseudonyme le « prénom sénégalais » qu'on lui avait attribué lors de son séjour à Dakar :

De l'ile de Gorée à Dakar / Pour tout le peuple noir / Petit Mbao ${ }^{32} /[\ldots]$ Afri-

30. Hip-Hop Paris-dakar, sortie en mai 2002, projet reconduit à Dakar en juillet 2002.

31. Bien qu'une grande partie de la chanson Descendant d'esclave soit intégrée sous forme de «featuring » (participation) de JK et de Béozedzed à une chanson commune figurant sur la mix-tape, Rage in fire. Par ailleurs, le texte collectif des Français, Quotidien, et la chanson de Dyara, Peuple noir, ont été composés pour ce projet.

32. Petit Mbao : banlieue de Dakar où résidaient les jeunes Français. 
caine je kiffe mes racines / [...] Dakar, Petit Mbao... un voyage si beau / Salaam Haleikhum à mes frères / à toutes mes sœurs soyez fières d'être noires, de couleur / Inch' Allah qu'on se retrouvera.

- par la mention de caractéristiques ethniques sénégalaises qui permettent au discours de JK dans la chanson Descendant d'esclave, d'ancrer le thème de l'esclavage dans une évocation plus générale d' "africanité » :

Douleur pour mes frères de couleur / Douleur du wolof au pulaar.

- par un hommage indirect, systématiquement renouvelé au difficile statut d'immigré de ses parents, originaires du Mali, dans les textes composés ou repris par Béozedzed suite à ce voyage (voir exemples 5 et 10).

Au-delà du contexte de cet atelier et de cet échange, il nous semble important de noter que le rap tire désormais sa légitimité du fait qu'il a très largement investi les espaces socio-culturels, socio-éducatifs, mais aussi médiatiques et économiques liés à la création musicale. Présent au sein des grands labels de production, largement diffusé sur les ondes radiophoniques et bien représenté par un certain nombre de magazines spécialisés, le rap est aussi très vivant dans les circuits plus underground de l'auto-production et de manifestations culturelles plus localisées.

Ainsi, et quelles qu'en soient les conséquences sur la teneur et la portée de ce discours, ou sur le phénomène de ghettoïsation, de simplification ou de « repli sur soi ${ }^{33}$ qu'il pourrait générer, le rap a désormais droit de cité au sein de nombreuses instances officielles, publiques ou privées. Dans le même temps, un certain nombre de ses représentants se sont dotés de moyens autonomes de production et de diffusion. Ce faisant, il nous semble que sur le marché culturel et langagier, le rap et ses acteurs ont acquis un capital symbolique influent. Formes, contenus et comportements associés y construisent une relation originale aux espaces et aux voix (voies!) du pouvoir. En outre, l'investissement du médium populaire et universel que constitue la chanson inscrit le discours rap dans un jeu de positionnements qui dépasse la problématique socio-culturelle et identitaire, et le projette dans la dimension de la création et de l'engagement, même paradoxal, inabouti ou, quelquefois, opportuniste ${ }^{34}$.

33. M. Boucher, 1998, p. 367.

34. Voir les critiques exprimées par certains rappeurs dans un numéro de la revue Radikal, Le magazine du mouvement hip-hop consacré au « réveil du rap militant » après le premier tour de l'élection présidentielle française en avril 2002 ( $\mathrm{n}^{\circ} 63$, juin 2002). 


\section{Quelle identité? Une nouvelle énonciation politique}

La portée politique des chansons rap de notre corpus ne relève pas uniquement d'une simple mise en pratique textuelle de positions élaborées en amont, soit au sein d'une scène plus globale qui pourrait se lire dans l'histoire du hip-hop, soit dans le cadre social, économique et linguistique que pourrait constituer « la banlieue ».

Bien sûr, on peut retrouver dans les textes des unités lexicales qui manifestent les spécificités morphologiques propres au français parlé dans les « cités » d'Ile-de-France. Voici quelques exemples tirés de nos corpus :

- néologie lexicale par verlanisation : «Du passé qui est encore zenpré » (Descendant d'esclave); "Je suis dans mon block je rap et je raconte au cromi » (Je suis dans mon block, je rap...); " Appelle le tiequar dis leur qu'en 2001 [...] On tchatche sur l'instrumental des lyrics ieuser » (Descendant d'esclave);

- néologismes synthématiques intégrant emprunt et verlan : «Kainfindies »;

- aphérèse : «c'est quoi son blème » (Descendant d'esclave),

- apocope : «Et si je ne suis pas d'accord je le dit au Mic» (Je suis dans mon block, je rap...), usage des sigles : «L'arme c'est la RAD et JK c'est la gachette » (RAD : Rébellion des Ancêtres par la Descendance; JK : nom du rappeur, Crie mon nom);

- emprunts fréquents à l'anglo-américain, souvent attestés dans le parler du groupe de pairs : «Les story de la vie des téci » (Je suis dans mon block, je rap...);

- technolecte : «[...] en rappant sur un bit», emprunts à d'autres langues que l'anglais comme le créole ou même couplet entier d'une chanson plurilingue rappé en créole par un rappeur particulier et dans lequel peuvent s'insérer des emprunts à l'anglais ou au français : «Pon moun pé fuck mwen! / Ou ja konèt mwen / on doug à fanm cé pou sa yo ka krie mwen rek 1 / [...] Apwesent ka pwen mic là, pou cote' mwen ka monté en tchouw! / Crie mon nom! [...] » (rappé par Rek 1, nom - jeu de mot écrit, Crie mon nom). Au niveau syntaxique, on notera par exemple les fréquentes constructions parataxiques : «Bateau, bataille, défaite brutale / Peuple éclaté fouetté / Besoin vital écarté / Mental parti, espoir fouttu / Douleur pour mes frères de couleur [...]. » (Descendant d'esclave). 
Ces propriétés morphologiques et syntaxiques sont autant de marqueurs linguistiques qui pourraient permettre de manifester une identité dont les chansons seraient le témoignage et la revendication : jeunes des cités issus de l'immigration... Or, si ces propriétés morphologiques et syntaxiques sont à remarquer, c'est bien parce qu'elles participent d'un processus identitaire d'individuation et de différenciation linguistique visà-vis d'un groupe auquel se voit rattaché en retour un français "standard » ou « socialement normé », porteur de représentations qui seraient refusées ou dénigrées. Mais il nous parait absolument nécessaire de ne pas nous en tenir à ces approximations : le positionnement énonciatif (et donc politique) des chansons mobilise certes des places discursives stéréotypées, mais les textes témoignent pour nous d'un travail plus profond, et aussi plus subversif.

\section{Micro-genres et mouvements discursifs}

L'analyse de l'énonciation représentée doit se faire pour chaque chanson; en effet il est clair que leur appartenance au sous-genre "chanson rap » n'assure pas une homogénéité énonciative. On notera, d'un point de vue théorique, qu'un genre textuel ne saurait constituer une essence. Chaque texte possède un mode d'appartenance générique qui doit être précisé. Un texte peut relever à la fois de la biographie, de la poésie, du pamphlet revendicatif (c'est le cas de la chanson Je suis dans mon block, je rap : «En 2000 je mange je dors mais je ne suis pas encore d'accord »). Cela constitue son pôle générique. La relation des éléments constituant ce pôle va produire un effet, qui peut être inédit, ce résultat est le produit de la matrice générique mise en œuvre ${ }^{35}$. Dans ce cadre, notre approche de l'énonciation représentée dans les textes tentera de préciser deux points à partir d'exemples précis tirés de notre corpus.

Premièrement, outre le découpage éventuel d'une chanson en terme de couplet/refrain, nous tentons d'analyser une chanson comme un ensemble de micro-genres hétérogènes, constitutifs de la cohésion et de la cohérence du texte. Le terme de micro-genre peut s'entendre dans un sens proche de la « séquence textuelle » proposée par J.-M. Adam ${ }^{36}$ mais nous

35. Pour ces concepts, appliqués à un corpus très différent, voir M. Barroco, V. Fayolle, 2001, « Mondes souterrains, légendes urbaines et méta-destination : vers une dynamique des genres narratifs », dans Sociétés - Revue des sciences humaines et sociales, 73, 3, De Boeck - Université, p. 87-98.

36. J.-M. Adam, 1997 ( $3^{\mathrm{e}}$ éd.), Les textes, types et prototypes, Paris, Nathan. 
préférons insister sur les concepts de «jeu de langage » et d' " air de famille » proposés par L. Wittgenstein ${ }^{37}$ que sur le concept de « prototype », issu de la psychologie cognitive et qui oblige à postuler "le meilleur exemplaire » d'un ensemble descriptif ou narratif... Un microgenre renvoie pour nous à une unité sémiologique de mode de donation des objets du discours ${ }^{38}$. Ce sont les mouvements entre les micro-genres déterminés d'une chanson qui vont caractériser l'énonciation. Par exemple, dans la partie chantée par JK de la chanson Descendant d'esclave on peut distinguer un micro-genre narratif illustrant la capture d'esclaves en Afrique :

Vie de rêve jusqu'à ce que les problèmes viennent / Prédateurs blèmes règnent et mon peuple saigne / Éloigné de la terre natale / Bateau, bataille, défaite brutale $[\ldots]$.

À la suite de ce premier ensemble on trouve un autre micro-genre composé d'une suite de questions qui constitue à la fois une mention réflexive de l'énonciateur à propos des évènements de son récit et un geste de destination vers un destinataire explicité par la dimension dialogale propre à la modalité phrastique de l'interrogation. Ce destinataire n'est pas spécifié mais il est censé être en connivence avec l'énonciateur pour que les questions posées manifestent une forme de rébellion :

Terre riche pillée, est-ce le prix à payer? / Est-ce que mon peuple doit plier? / Ou est-ce qu'il faut se réveiller?

Le passage de JK se termine par l'énoncé de sa propre situation, ce qui constitue un troisième micro-genre :

J'suis le sang de leur sang / La rage au sang / Descendant d'homme noir souffrant / C'est... que je vis parmi les français.

Le premier micro-genre permet d'installer une première scène que l'on pourrait appeler à la suite de D. Maingueneau ${ }^{39}$ une « deixis fondatrice » : la relation « prédateurs blêmes »/ « mon peuple ». Le second micro-genre

37. L. Wittgenstein, 1961, Investigations philosophiques (trad. franç.), Paris, Gallimard.

38. F. François, 1994, « Langage, sémiotiques, mondes, dialogisme, interprétation », dans Cahiers de l'institut de linguistique de Louvain, 73, Louvain-la-Neuve, p. 239-272.

39. D. Maingueneau, «Ethos, scénographie et incorporation », dans R. Amossy (dir.), 1999, Images de soi dans le discours. La construction de l'éthos, Lausanne, Delachaux \& Niestlé, p. 75-100. 
va introduire un pont entre le récit d'une situation passée et une situation présente et le troisième micro-genre va pouvoir exposer, instituer, toute la difficulté et l'ambigüité de la position énonciative en s'appuyant sur la légitimité apportée par la « deixis » fondatrice : «C'est... que je vis parmi les français ». Le fait que le premier micro-genre soit un récit n'est pas un hasard : il introduit une trame qui fonde une origine que l'énonciation pose et par laquelle elle se reconnait. Le positionnement politique s'effectue ainsi en faisant intervenir une histoire présentée comme fondatrice au sein du champ social, sans pour autant qu'il existe de solutions déjà élaborées, une issue déterminée, comme cela peut être le cas dans une démarche révolutionnaire ou même dans la justification d'une action politique au nom d'une utopie reconnue comme telle mais assumée comme « télos » directeur. Ceci explique sans doute cette relative (ce n'est en effet pas vrai pour tous les groupes) retenue envers un engagement politique « classique » (partis, associations...) de la part des rappeurs ${ }^{40}$ ainsi que la difficulté inhérente à la réception politique des textes rap.

\section{Une nouvelle voix du français}

Deuxièmement, il nous a semblé qu'une lecture de certains textes de M. Bakhtine pouvait permettre de mieux comprendre le jeu des représentations engagées dans les chansons. En effet, à la fin de l'Esthétique de la création verbale $^{41}, \mathrm{M}$. Bakhtine oppose ce qui dans un texte relève de la « langue » et ce qui relève du «particulier ». Selon lui, le premier point désigne tout ce qui est de l'ordre du reproductible, de la seule « mise en parole » du code, de tout ce qui existe par ailleurs hors du texte, dans d'autres occurrences discursives de la même «langue ». Le second point désigne ce qui est unique, non reproductible. Il relie le texte considéré non pas aux éléments reproductibles d'un système linguistique mais aux autres textes en un rapport « dialogique ». Dans leur définition, ces pôles ne peuvent s'expliciter qu'en s'opposant, l'un étant du registre de la « réification », l'autre étant du registre du « dialogisme ». Pour nommer leur

40. Sur ce point Musique et politique. Les répertoires de l'identité, voir M. Souchard, dans A. Darré (dir.), 1996, La différence rap, Rennes, PUR, p. 257-263. On peut lire aussi l'interview de MADJ, rappeur du groupe Assassin (exemple : «Je crois que la notion de mouvement de contre-culture, concernant le rap français n'existe pas en fait : un mouvement suppose de se donner les moyens de réagir, collectivement, et de façon très autonome quand il le faut »), dans Radikal, Le magazine du mouvement hip-hop, 2002, p. 10.

41. M. Bakhtine, 1978, "Le problème du texte », dans Esthétique de la création verbale (trad. franç.), Paris, Gallimard. 
relation M. Bakhtine propose, un peu plus loin, l'application du « principe de complémentarité ${ }^{42}$. Il faut bien saisir que ce terme de « complémentarité » ne désigne nullement un rapport formant une totalité, une complétude, mais renvoie au principe élaboré par le physicien Niels Bohr dans le cadre de la physique quantique, et qui désigne au contraire une " exclusion mutuelle » de deux manifestations d'une même « réalité » qui se distribuent selon des protocoles expérimentaux différents : « la relation d'exclusion désignée par le concept de complémentarité caractérise des termes qui seraient contradictoires s'ils étaient réunis dans une même description ${ }^{43}$. La tentative de M. Bakhtine pour dépasser cette situation consiste à comprendre le rapport de complémentarité lui-même « de manière dialogique ». L'exclusion mutuelle posée par le principe de complémentarité est comprise alors comme une forme de polyphonie dont une voix serait la/une langue, comprise comme un concept non pas «neutre » ou « objectif» mais impliquant une image de relation à l'autre. Jouer avec cette image, c'est donc déterminer à nouveau une scène énonciative. Le concept de voix, par lequel se résout le rapport de complémentarité, implique l'existence de «représentations» qui se font entendre par l'usage même d'une (ou plusieurs) langue. On peut penser que cette intuition avait été préparée par les analyses que M. Bakhtine avait proposées lors du troisième chapitre de l'ouvrage Esthétique et théorie du roman (chapitre intitulé «Le plurilinguisme dans le roman »). En effet, à partir des relations hypertextuelles, M. Bakhtine saisit l'entrelacs des voix caractéristiques des registres, propres à certains genres (comme l'éloquence juridique, le compte rendu des séances du Parlement...), qui intègrent un texte relevant du genre $d u$ « roman humoristique ». La relation d'intégration s'entend alors comme parodie ou pastiche. De ce fait on entend, pour le même passage, deux perspectives qui s'entrecroisent dans ce que M. Bakhtine appelle une structure « hybride » comportant deux « accents » différents.

Il nous semble que les chansons rap vont utiliser cette structure hybride afin de mobiliser non pas tant des genres différents que des intervenants qui vont pouvoir prendre la parole à l'intérieur même d'un discours clairement organisé par un pôle énonciatif dominant :

42. M. Bakhtine, «Remarques sur l'épistémologie des sciences humaines », dans Esthétique de la création verbale.

43. N. Bohr, 1991, Physique atomique et connaissance humaine, (trad. franç.) Paris, Gallimard. 
Enfermé, privé de liberté ça veut dire quoi ça! / Je viens sur le bit fầché, les gens vont dire c'est quoi son problème encore. (Beozedzed, Descendant d'esclave)

Ma vision des choses c'est ce que je pose sur le bit / Pas rose eh ouai qu'est-ce que t'en dis c'est pas ma faute si c'est pourri / T'as vu c'est resté constant donc pas le temps de faire l'endormi / Prévient tous tes amis et puis bien fort tu leurs dis / « Eh! si on se regroupe tous comme dans un concert/même si plein de truc de ouf foutent nos projets en l'air» / moi on me dit qu'il y a plus de 36 manières $[\ldots]$.

Ce jeu énonciatif pluralise les positionnements et rend complexe l'assignation du texte à un point de vue. La mise en scène du divers permet à la fois de caricaturer un point de vue que l'on juge néfaste, de faire apparaitre l'aspect pluriel du groupe de pairs, d'anticiper sur des réceptions imaginaires.

Revenons alors à la voix linguistique telle que la propose M. Bakhtine. La langue, objet social s'il en est, va alors se retrouver munie de valeurs. Une chanson rap peut ainsi convoquer en contexte certaines représentations attachées au français et intervenir sur elles. Les procédés morphologiques de modification de mots français existants impliquent par exemple une modification des rapports au français. Dans nos textes, c'est la corrélation de ces procédés avec l'inscription d'une trame historique inédite pour une certaine forme d'identité "française» (se reconnaitre des ancêtres esclaves par exemple) qui va opérer un positionnement politique d'un nouvel ordre. Les procédés morphologiques, les emprunts, ne signifient pas par eux-mêmes mais ils participent à une stratégie de modification du sens des mots élaborée par le travail du texte. Ce travail des mots modifie la voix du français, ne sort pas du français. Même les longs passages en créole inscrivent la voix du créole en la tissant avec le français et, de ce fait, en modifient encore la voix. L'espace communautaire dans lequel, rappelons-le, la jeunesse citadine issue souvent de parents immigrés est censée «s'intégrer» ne saurait se penser selon les rappeurs comme un espace pré-formé dans lequel ils devraient se glisser, discrètement si possible. Mobilisant certains aspects de la voix du « français » ils s'inscrivent dans ses inflexions, modifient sa portée.

Dans un article précédent ${ }^{44}$, nous avions montré comment le rap se sert des variations morphologiques pour modifier le sémiotisme même des lexèmes. Ceux-ci devenaient en effet des mots-effets porteurs d'attitudes

44. M. Auzanneau, M. Bento, V. Fayolle, 2002, p. 90-91. 
énonciatives singulières. Cette dimension rhétorique, énonciative et sémantique permet de faire entendre d'autres représentations dans «le français ». En insistant sur la dimension corporelle du pôle énonciatif, de l'éthos ${ }^{45}$, on peut suivre D. Maingueneau parlant du «ton » d'une énonciation puisque c'est un nouveau ton, une nouvelle attitude qui se fait entendre en français et qui, en même temps, ne se reconnait pas « naturellement » française :

Je regarde à l'horizon, je vois qu'il y a des choses qui se font / des groupes qui témon de bonnes connexions / Eh qu'est ce que t'en dis si on faisait pareil qu'eux / Allez allons-y représentons aussi pour nos possee! (Beozedzed, Je suis dans mon block, je rap...)

Dans cet exemple, l'emprunt au rap américain «possee » permet certes de manifester son appartenance au mouvement hip-hop mais, d'une part, s'ancre dans une réalité sociale spécifique qui n'est plus celle des ghettos noirs américains et, d'autre part, fait entendre une forme d'affect, d'affinité (qu'on n'entend plus dans la traduction par « groupes », « clans » ou « amis ») que l'interprète-auditeur de la chanson doit saisir, à condition d'opérer ce que l'on peut appeler un mouvement de décentrement, tout à la fois linguistique, corporel et intellectuel, qui laisse place alors à un nouvel ordonnancement de l'espace public.

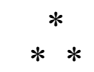

Dans cette relation entre la production et la réception d'un texte apparait une autre voix du français qui opère une modification, pour peu qu'elle soit entendue. Cette forme de modification, fragile (aucun des pôles de cette scène ne la représente ou ne peut se l'accaparer), pourrait être la forme la plus fondamentalement politique du rap. La langue, dans sa triple dimension systémique, symbolique et sociale, y est le lieu en même temps que l'enjeu privilégié d'une élaboration stylistique critique (dénonciation, revendication, témoignage...), ancrée dans la mise en scène de réalités sociales prégnantes. Cette dernière permet aux rappeurslocuteurs-acteurs de ces réalités d'exposer les formes et conditions particulières de leur appropriation et de leur ré-élaboration des normes de

45. D. Maingueneau, 1999, p. 75-100. 
communication et de comportement a priori socialement partagées, ainsi que des représentations, des positionnements et des catégorisations qui leur sont associés. Cette appropriation a lieu et se donne à voir à travers un ensemble d'attitudes apparemment paradoxales, allant du respect de ces normes à leur rejet, et de leur application rigoureuse à la production de pratiques et de modèles nouveaux, endogènes, ostentatoires.

\section{Bibliographie}

ADAM J.-M., 1997 (3éd.), Les textes : types et prototypes, Paris, Nathan Université.

ANDROUTSOPOULOS J., SCHOLZ A, 1999, On the recontextualization of hiphop in european speech communities a contrastive analysis of rap lyrics, Americanization and popular culture in Europe, Conférence internationale Ascona (CH), November 10-14, 1999. Document électronique disponible à http://www.archetype.de/hiphop/ascona.html.

AUZANNEAU M., Bento M., Fayolle V., Lambert P., Trimaille C., Amar L., Fernandes A., 2000, « Le rap en France et ailleurs : intérêt d'une démarche pluridisciplinaire », communication au colloque de Tours, France : pays de contact de langues, octobre 2000.

- 2001a, «Identités africaines, le rap comme lieu d'expression », dans Cahiers d'Études Africaines, 163-164, XLI-3-4, sous la direction de C. Canut, Paris, EHESS, p. 711-734.

— 2001b, «Le rap, expression de dynamiques urbaines plurilingues : le cas de Libreville », dans Plurilinguismes, Le plurilinguisme à Libreville, 18, p. 11-48.

- Avec Bento M., Fayolle V., 2002, «De la diversité lexicale dans le rap au Gabon et au Sénégal », dans La Linguistique, vol. 38, fasc. 1, p. 69-98.

BAKHTINE M., 1978 (trad. franç.), Esthétique et théorie du roman, Paris, Gallimard.

BENVENISTE E., 1974, «L'appareil formel de l'énonciation », dans Problèmes de linguistique générale, Paris, Gallimard, tome 2, p. 79-88.

BILLIEZ J., 1998, "L'alternance des langues en chantant», dans LIDIL, 18, " Alternance des Langues et enjeux identitaires », p. 125-140.

- 1996, "Poésie musicale urbaine : langues et identités entrelacées », dans L.-J. Calvet, C. Juillard (dir.), Actes du Colloque de Dakar, Les politiques linguistiques, mythes et réalités, p. 61-66.

BOUCHER M., 1998, Rap, expression des lascars. Significations et enjeux du rap dans la société française, Paris, L'Harmattan. 
BOURDIEU P., 1982, Ce que parler veut dire, Paris, Fayard.

BRES J., 1993, «Le jeu des ethnosociotypes », dans Plantin (dir), Lieux communs : topoï, stéréotypes, clichés, Paris, Kimé, p. 152-161.

CALVET L.-J., 1994, Les voix de la ville, Introduction à la sociolinguistique urbaine, Paris, Payot.

CASOLARI F., 1999, « Constructions stéréotypiques dans le rap marseillais », dans Gasquet-Cyrus M., Kosmicky G., Van den Avenne C. (dir.), Paroles et musiques à Marseille, Paris, L'Harmattan, p. 73-92.

FRANÇOIS F., 1993, Pratiques de l'oral, dialogue, jeu et variations des figures du sens, Paris, Nathan.

JACONO J.-M., 1999, « Le rap : une introduction », dans Gasquet-Cyrus et al., p. 25-56.

LE PAGE R.-B., TABOURET-KELLER A., 1985, Acts of identity, Creole Based Approach to language and ethnicity, Cambridge, Cambridge University Press.

MAINGUENEAU D., 1999, «Ethos, scénographie, incorporation », dans Amossy R. (dir.), Images de soi dans le discours. La construction de l'éthos, Neuchâtel, Delachaux et Niestlé.

MILROY L. \& J., 1990, Social network and social class : towards an integrated sociolinguistic model, Des langues et des villes, Actes du colloque de Dakar, ACCT, Paris, Didier Érudition, p. 97-114.

MONDADA L., 2000, Décrire la ville, Bruxelles, Anthropos (diff. Paris, Economica).

MYERS-SCOTTON C., 1983, « The negociation of identities in conversation : a theory of markedness and code choice », International Journal of Sociology of Language, 44, p. 115-136.

NICOLAÏ R., 2000, « La construction de l'unitaire et le « sentiment de l'unité » dans la saisie du contact des langues », Communication au colloque du LACIS Langues en contact et incidences subjectives, 16-17 juin 2000, Montpellier, Version provisoire du texte publié en 2001 dans Traverses, 2.

- 2001, «Exploration dans l'hétérogène : miroirs croisés », Cahier d'Études africaines, 163-164, XLI-3-4, p. 309-321.

TRIMAILLE C., 1999 a, De la planète mars... codes, langages, identités : étude sociolinguistique de textes de rap marseillais, DEA, sous la direction de J. Billiez, Université Stendhal - Grenoble 3.

- 1999 b, « Le rap français ou la différence mise en langues », dans LIDIL, 19, «Les parlers urbains », p. 79-98. 